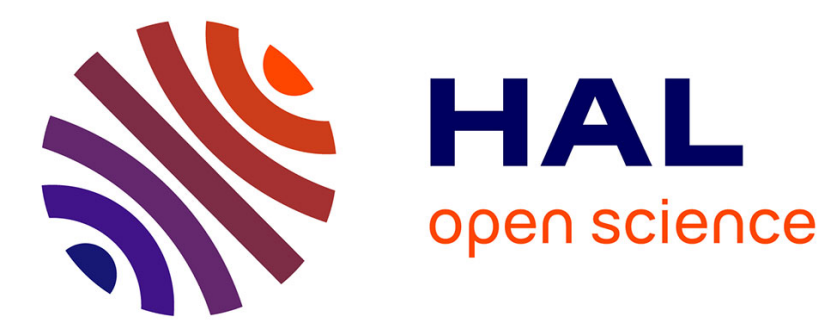

\title{
Macédoine, le bilan en demi-teinte de la politique européenne
}

Nadège Ragaru

\section{To cite this version:}

Nadège Ragaru. Macédoine, le bilan en demi-teinte de la politique européenne: Le bilan en demiteinte de la politique européenne. Critique Internationale, 2004, 24, pp.9-20. 10.3917/crii.024.0009 . hal-01020116

\section{HAL Id: hal-01020116 https://hal-sciencespo.archives-ouvertes.fr/hal-01020116}

Submitted on 7 Jul 2014

HAL is a multi-disciplinary open access archive for the deposit and dissemination of scientific research documents, whether they are published or not. The documents may come from teaching and research institutions in France or abroad, or from public or private research centers.
L'archive ouverte pluridisciplinaire HAL, est destinée au dépôt et à la diffusion de documents scientifiques de niveau recherche, publiés ou non, émanant des établissements d'enseignement et de recherche français ou étrangers, des laboratoires publics ou privés. 


\section{Macédoine : le bilan en demi-teinte de la politique européenne}

Presses de Sc. Po. | Critique internationale

2004/3 - no 24

pages 9 à 20

ISSN 1290-7839

Article disponible en ligne à l'adresse:

http://www.cairn.info/revue-critique-internationale-2004-3-page-9.htm

Pour citer cet article :

"Macédoine : le bilan en demi-teinte de la politique européenne ", Critique internationale, 2004/3 no 24, p. 9-20.

Distribution électronique Cairn.info pour Presses de Sc. Po..

(c) Presses de Sc. Po.. Tous droits réservés pour tous pays.

La reproduction ou représentation de cet article, notamment par photocopie, n'est autorisée que dans les limites des conditions générales d'utilisation du site ou, le cas échéant, des conditions générales de la licence souscrite par votre établissement. Toute autre reproduction ou représentation, en tout ou partie, sous quelque forme et de quelque manière que ce soit, est interdite sauf accord préalable et écrit de l'éditeur, en dehors des cas prévus par la législation en vigueur en France. II est précisé que son stockage dans une base de données est également interdit. 


\title{
Contre-jour
}

\author{
par Nadège Ragaru
}

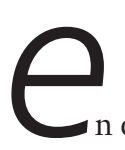

cette période de remise en question de l'action internationale au Kosovo, le retour à la paix en Macédoine reste la principale success story de la politique européenne dans les Balkans. Oublié le temps où les Européens, paralysés par le veto de la Grèce, hésitaient à reconnaître l'indépendance de l'État de Macédoine, né de l'éclatement de la Yougoslavie''. Oubliée l'époque où, toute à la contemplation de cette « oasis de paix » balkanique, l'Union européenne négligeait de soutenir la démocratisation du pays et d'encourager une meilleure représentation de la minorité albanaise (environ un quart de la population²) au sein de l'appareil d'État et des forces de l'ordre. Lorsqu'en février 2001, l'émergence d'une guérilla albanaise à la frontière avec le Kosovo donne à craindre l'éclatement d'une nouvelle guerre dans les Balkans, l'Union européenne comprend l'urgence d'une intervention ${ }^{3}$. Elle accepte également de travailler de concert avec les États-Unis pour permettre la signature d'un accord de paix à Ohrid, le 13 août 2001.

Depuis lors, c'est à la mise en œuvre des changements législatifs et institutionnels prévus par le compromis d'Ohrid que se consacrent l'Office du Représentant

\footnotetext{
1. La Grèce accusait la Macédoine d'avoir usurpé un symbole grec, le soleil de Vergina, pour son drapeau et d'avoir adopté un nom appartenant au patrimoine culturel et historique grec. Elle s'inquiétait également de voir la Constitution macédonienne servir de prétexte à l'exercice d'un droit de regard macédonien sur les slavophones de Macédoine égéenne. C'est finalement sous le nom de FYROM (Former Yugoslav Republic of Macedonia) que la Macédoine a été admise à l'ONU en avril 1993. Les États membres de l'Union européenne ont reconnu l'indépendance du nouvel État en ordre dispersé au printemps 1994. En dépit de l'organisation des négociations entre la Macédoine et la Grèce sous l'égide des Nations unies à New York, la querelle du « nom » n'est toujours pas résolue.

2. Selon les résultats du dernier recensement conduit en novembre 2002 et supervisé par la communauté internationale, les Albanais représentent 25,17 \% d'une population de 2,022 millions d'habitants. Ils étaient 22,67 \% en 1994. La part des citoyens de Macédoine s'identifiant comme ethniquement macédoniens est passée de 66,60 \% en 1994 à 64,18 \% en 2002. Parmi les autres minorités, on notera la présence de Turcs (3,85\% en 2002), de Roms (2,66 \%) et de Serbes (1,78\%).

3. Sur les événements de 2001, voir Nadège Ragaru, « Dits et non-dits de la crise macédonienne », Politique internationale, 92 , été 2001, p. 165-201.
} 
spécial de l'Union européenne en Macédoine et le gouvernement modéré issu des élections générales de septembre 2002, une coalition entre l'Alliance social-démocrate (SDSM, centre-gauche) de Branko Crvenkovski et l'Union pour l'intégration démocratique (BDI), que dirige l'ancien leader des rebelles albanais, Ali Ahmeti. Présente sur le terrain politique et économique par le truchement du Processus de stabilisation et d'association (PSA) ${ }^{4}$, l'Union européenne a également endossé d'importantes responsabilités dans le domaine de la sécurité et des affaires intérieures : en mars 2003, une première mission militaire européenne, Concordia, a pris la relève de l'OTAN. Six mois plus tard, elle a été remplacée par Proxima, une opération de police forte de quelque deux cents hommes. Au début du mois de juin 2004, l'Alliance atlantique a par ailleurs annoncé le départ des derniers soldats américains présents sur le sol macédonien. L'Union européenne le souhaitait : elle constitue désormais le principal acteur international en Macédoine avec, à charge, la pérennisation de la paix et la réalisation des perspectives d'intégration réaffirmées lors du Sommet Union européenne-Balkans, le 21 juin 2003, à Thessalonique.

L'enjeu est d'envergure même si le bilan des efforts internationaux déployés depuis 2001 peut, de prime abord, paraître positif. Désarmée, l'ancienne guérilla albanaise a formé en juin 2002 un parti politique, l'Union pour l'intégration démocratique, qui soutient le retour à une coexistence intercommunautaire apaisée. De son côté, l'Alliance de B. Crvenkovski est convaincue que le seul moyen de sortir le pays de «l'ornière balkanique » est de trouver un compromis ethnique, condition sine qua non d'une entrée dans l'Union européenne et dans l'OTAN5. Le fait que la Macédoine ait déposé sa candidature à l'UE le 22 mars dernier, alors même que certains observateurs occidentaux avaient suggéré un report de calendrier, souligne l'importance stratégique que les autorités accordent à cet objectif. Enfin, dernière « preuve » de réussite du processus de stabilisation sous supervision de la communauté internationale, la Macédoine a géré avec calme deux événements dont on aurait pu craindre qu'ils ne fassent dérailler le processus de paix : le décès accidentel du Président Boris Trajkovski, le 26 février 20046, et les affrontements intercommunautaires des 17-18-19 mars 2004 au Kosovo voisin?

\footnotetext{
4. La Macédoine a signé un Accord de stabilisation et d'association (ASA) avec l'Union européenne le 9 avril 2001. L'Accord a été ratifié le 23 février 2004.

5. La Macédoine avait fait connaître son désir d'intégrer l'OTAN lors du Sommet de Prague de novembre 2002. En mai 2003, une Charte adriatique, réunissant trois États des Balkans de l'Ouest qui souhaitaient rejoindre l'Alliance - l'Albanie, la Croatie et la Macédoine -, a été mise en place avec le soutien des États-Unis.

6. L'élection présidentielle anticipée des 14 et 28 avril 2004 a été remportée par le Premier ministre, Branko Crvenkovski (SDSM), qui a cédé son poste à Hari Kostov, auparavant ministre de l'Intérieur.

7. Suite à la diffusion par la télévision kosovare, le 16 mars, d'informations selon lesquelles trois enfants albanais originaires du village de Çabër/Čabra (municipalité de Zubin Potok, au nord du Kosovo), pourchassés par des enfants serbes, seraient morts noyés dans la rivière Ibar, de violents affrontements intercommunautaires ont éclaté entre Albanais et représentants
} 
S'en tenir à cette lecture trop lisse des évolutions en Macédoine serait pourtant une erreur. Car le pari fait par les élites macédoniennes est pour le moins osé. Le rééquilibrage des rapports intercommunautaires - accepté à contrecœur par la majorité macédonienne-slave - intervient dans un contexte économique et social extrêmement préoccupant. Les réformes requises par la restructuration de l'économie et la préparation de l'intégration européenne ont un coût social élevé et alimentent des frustrations qui pourraient se manifester dans un registre identitaire. Combien de temps faudra-t-il avant que le mécontentement sensible dans la société ne trouve une traduction politique et quelle forme cette dernière prendra-t-elle ? Voici deux interrogations auxquelles il paraît urgent de répondre si l'on souhaite ajuster les dispositifs européens aux besoins de la Macédoine et confirmer le rétablissement de la paix.

\section{Où en est-on dans la mise en œuvre des accords d'Ohrid ?}

Le premier volet de l'action européenne en Macédoine concerne la mise en œuvre des accords d'Ohrid supervisée par le Représentant spécial de l'Union européenne en Macédoine, poste occupé depuis le 1 1 er août 2004 par le diplomate suédois, Michael Sahlin. L'objectif est de parvenir à clore la transposition juridique et institutionnelle des accords d'ici 2005-2006. À cette date, l'Office du Représentant devrait fermer ses portes pour ne laisser en Macédoine que l'Agence pour la reconstruction européenne (AER) et une Délégation de la Commission européenne au rôle renforcé. Si l'on s'en tient à la lettre des accords, la perspective est sans doute réaliste. Mais la question est ailleurs : les accords d'Ohrid suffiront-ils à garantir la stabilité de la Macédoine et quels sont leurs effets sur les rapports entre majorité slave et minorité albanaise ?

Pour mettre un terme à six mois d'affrontements entre les forces de l'ordre macédoniennes et des rebelles albanais rassemblés dans l'Armée de libération nationale (Ushtria Çlirimtare Kombëtare, UÇK), le compromis d'Ohrid avait prévu de renforcer les mécanismes de protection des droits de la communauté albanaise en échange du démantèlement de la guérilla et de la réaffirmation de l'intégrité territoriale de la Macédoine. Concentrés pour l'essentiel dans l'Ouest et le NordOuest du pays, les Albanais souhaitaient une plus grande reconnaissance linguistique et culturelle, une représentation équitable dans la fonction publique, un

\footnotetext{
de la minorité serbe, dans la région de Mitrovica et à travers l'ensemble du Kosovo. En trois jours, ces violences auraient fait 19 morts, 954 blessés et 4100 déplacés selon les chiffres de l'OSCE. Par ailleurs, quelque 550 maisons et 27 églises et monastères orthodoxes ont été détruits. Il s'agissait de la pire flambée de violence dans cette province sous contrôle international depuis juin 1999. Chiffres cités dans OSCE Mission in Kosovo, Human Rights Challenges Following the March Riots (http://www.osce.org/documents/mik/2004/05/2939_en.pdf) (consulté le 17 septembre 2004)
} 
accès à l'enseignement supérieur en albanais ainsi qu'une décentralisation de la gestion territoriale ${ }^{8}$. Sur ces divers points, ils ont été pour l'essentiel entendus. La communauté internationale - et particulièrement Alain Le Roy, alors envoyé spécial de l'Union européenne en Macédoine - ont dû user de toute leur influence auprès du gouvernement pour garantir l'adoption, le 16 novembre 2001, des amendements à la Constitution de 1991 prévus au titre des accords.

C'est sans doute dans le domaine sécuritaire que les progrès ont été les plus remarqués. Avant août 2001, la police macédonienne ne comprenait que $3 \%$ d'Albanais. Les responsables politiques de la communauté souhaitaient que des forces de police autonomes soient instaurées dans les régions où les Albanais étaient majoritaires. La police a finalement été maintenue sous le contrôle du ministère de l'Intérieur, mais les Albanais doivent y être proportionnellement représentés9 ${ }^{9} \mathrm{Au}$ 1er juillet 2003, environ 1065 policiers issus des minorités (principalement des Albanais, mais aussi quelques Turcs, Bosniaques, Serbes, Roms et Valaques) avaient été formés par les soins de l'OSCE. Dès 2002, des unités de police mixte ont été déployées dans les anciennes zones de conflit.

Si, lors des émeutes de mars 2004 au Kosovo, les régions de Macédoine à majorité albanaise sont restées stables, les crispations ne sont pas absentes pour autant. À Tetovo, les habitants slaves continuent à prendre le chemin de la capitale, poussés par la recherche d'un emploi, mais aussi, souvent, par la peur de se retrouver dans un univers albanais en voie d'homogénéisation. Preuve de la persistance des sentiments de défiance, une opération de collecte des armes illégales, menée en novembre-décembre 2003, a donné des résultats décevants ${ }^{10}$. Aujourd'hui encore, la police peine à remplir ses missions en zone albanaise et les tensions restent très vives lorsque les forces de police tentent d'arrêter des représentants des milieux illicites ayant eu partie liée avec l'UÇK.

Le second chantier ouvert par les accords d'Ohrid concernait la représentation des minorités dans la fonction publique. En 2001, les Albanais y occupaient environ

\footnotetext{
8. Les revendications albanaises ne datent pas de 2001. Elles sont apparues dans le sillage de la création d'un État de Macédoine indépendant en septembre 1991 et se sont accentuées au cours des années 1990 en partie sous l'effet des tensions observées au Kosovo voisin. Les Albanais souhaitaient notamment que soit introduit dans la Constitution le principe de l'égalité constitutive des peuples albanais et macédonien. Par ailleurs, le durcissement des relations entre majorité slave et minorité « majoritaire » albanaise a parfois placé dans une situation malaisée les autres communautés (rom, turque ou valaque, par exemple) de Macédoine. Cf. Christophe Chiclet, Bernard Lory (dir.), La République de Macédoine, Paris, L'Harmattan, 1998. 9. Au ministère de l'Intérieur, en octobre 2003, 8,1 \% des employés étaient albanais. À ces chiffres doivent être ajoutés les 500 Albanais ayant achevé leur formation en juillet 2003. Les communautés minoritaires restent absentes des forces de police spéciales. Dans l'armée, si des efforts ont été enregistrés pour accroître la présence d'Albanais, leur part dans les forces spéciales et le renseignement est infime. Cf. International Crisis Group, Macedonia: No Room for Complacency, octobre 2003, p. 4. 10. Sur les 600000 armes circulant illégalement dans le pays (estimations de l'UNHCR, 2003), seules 7500 ont été remises. Cet échec est également dû à l'opposition des maires du Parti démocratique albanais (PDA) à la collecte dans vingt-trois municipalités à majorité albanaise.
} 
$10 \%$ des emplois ${ }^{11}$. Trois ans plus tard, le bilan paraît nuancé. Si des nominations sont intervenues au sommet de l'État - le gouvernement actuel compte quatre ministres et un vice-Premier ministre albanais ; le vice-président du Parlement est albanais -, plus bas dans la hiérarchie, l'attribution de postes à des représentants des minorités a pris du retard ${ }^{12}$. Dans certaines instances, les lenteurs sont imputables aux réticences de responsables slavo-macédoniens ; mais, plus souvent, la difficulté provient du manque de cadres albanais et du caractère peu incitatif des salaires offerts dans l'administration. La nécessité de passer par des réseaux politiques pour obtenir un poste constitue parfois aussi un facteur dissuasif. Tout changement de majorité (au sein de la communauté albanaise) risque de se traduire par un licenciement. Aux yeux de ceux qui avaient trouvé à se réaliser dans le secteur privé, le jeu n'en vaut pas toujours la chandelle.

Troisième axe de réforme posé à Ohrid, l'enseignement supérieur. Un début de solution au problème de l'usage de la langue albanaise avait été trouvé à l'automne 2001 avec l'ouverture, à Tetovo, de l'Université pour l'Europe du Sud-Est, un établissement financé par l'Union européenne et où les enseignements sont dispensés en anglais, en albanais et en macédonien. Mais c'est autour de l'Université privée de Tetovo, créée en 1994 et non reconnue par les autorités macédoniennes, que les attentes albanaises se sont cristallisées. La légalisation de l'Université de Tetovo a finalement été obtenue en février 2004, au terme de débats politiques mettant en jeu non seulement les intérêts de la majorité macédonienne-slave, mais également ceux d'acteurs politiques locaux pouvant souhaiter entretenir un climat d'incertitude. Avec ses quatre facultés accréditées (sciences humaines, sciences naturelles, droit, économie), l'Université de Tetovo constitue désormais le troisième établissement d'enseignement supérieur public de Macédoine, après ceux de Skopje et de Bitola, et devrait bénéficier à ce titre de subsides publics.

Cette mesure, très symbolique, ne suffira pourtant pas à résoudre la crise de l'enseignement. L'éducation secondaire souffre d'un manque de moyens d'autant plus sensible que, depuis 2001, les parents des élèves des deux principales communautés sont de plus en plus réticents à laisser leurs enfants fréquenter le même établissement. En de nombreuses circonstances, un système de rotation dans l'usage des bâtiments a été introduit ; mais cet aménagement des horaires n'a pas toujours suffi à assurer le retour au calme. Par ailleurs, les expériences multiethniques tentées à Bitola et à Skopje à la rentrée scolaire de septembre 2003 ont échoué. De façon plus générale, on assiste à la mise en place d'un système d'enseignement dual qui alimente des représentations mutuelles négatives.

11. Cf. Suncica Stojanovska, « Macedonia: Albanians Still Underemployed », Balkan Crisis Report, 436, 10 juin 2003.

12. Le budget 2004 prévoit au total 600 recrutements de représentants des communautés minoritaires au sein de la fonction publique. Voir « 2004 Budget Passed», Macedonian Information Agency, 29 décembre 2003. 
La principale pomme de discorde reste cependant la politique de décentralisation : quatrième pilier d'Ohrid. L'accord du 13 août, complété par les amendements constitutionnels du 16 novembre 2001, a posé que toute langue parlée par plus de $20 \%$ de la population serait considérée comme langue officielle au niveau local et utilisable dans l'administration ${ }^{13}$. Par ailleurs, un nouveau découpage territorial devait être adopté et l'autonomie des municipalités renforcée. La délimitation des communes apparaît comme un enjeu politique majeur dans la mesure où elle conditionnera les rapports de force entre communautés. Là où les partis slaves semblent soucieux d'empêcher la formation de régions albanaises compactes - qui pourraient être, un jour, tentées de faire sécession -, les Albanais souhaitent éviter un découpage « en gruyère », enserrant de petites communes albanaises dans des ensembles slaves comme cela avait été le cas en 1992. Le 15 juillet 2004, le gouvernement a adopté un projet de loi sur le découpage territorial prévoyant de ramener le nombre total des municipalités de 123 à 80, puis 76 en 2008. À Skopje, le rattachement des communes à dominante albanaise de Saraj et de Kondovo devrait faire de l'albanais la seconde langue officielle. À Struga, les Albanais se retrouveraient en majorité après l'incorporation dans la nouvelle municipalité de communes rurales albanaises. En revanche, la délimitation de Kičvevo resterait inchangée contrairement au souhait des Albanais. L'annonce du projet a suscité des réactions extrêmement négatives dans l'opinion publique slave, parmi les représentants des minorités (turques et roms notamment), ainsi que dans l'opposition politique (l'Organisation révolutionnaire intérieure de Nikola Gruevski (VMRO-DPMNE, nationaliste) au pouvoir entre 1998 et 2001 et l'alliance « Troisième voie »). Dans la seconde moitié du mois de juillet 2004, les manifestations se sont succédé à Struga et à Skopje, faisant 17 blessés à Struga le 23 juillet, tandis que plus d'une quarantaine de municipalités soumettait la question du découpage municipal à des référendums locaux (aux résultats non contraignants). Les opposants au maillage communal proposé par le gouvernement ont finalement obtenu qu'un référendum sur la décentralisation soit organisé, le 7 novembre 200414. L'issue de la consultation

\footnotetext{
13. L’article de la Constitution de 1991 qui stipulait que « la langue macédonienne, écrite en utilisant son alphabet cyrillique, est la langue officielle de la République de Macédoine » a été amendé de la manière suivante : « La langue macédonienne, écrite en utilisant son alphabet cyrillique, est la langue officielle en République de Macédoine et dans les relations extérieures de la République. Toute autre langue parlée par au moins $20 \%$ de la population constitue également une langue officielle, écrite en utilisant son alphabet ». On notera par ailleurs que la règle des $20 \%$ ne concerne pas seulement la communauté albanaise, mais aussi les Serbes (dans la région de Kumanovo, par exemple) ou les Roms (ainsi, à Sutka, dans l'agglomération de Skopje). En revanche, les Albanais ne sont pas parvenus à obtenir le statut de « peuple constitutif » de Macédoine. 14. Au terme de l'article 73 de la Constitution de 1991, le Parlement est obligé de convoquer un référendum si 150000 citoyens ont signé une pétition en ce sens dans les six mois suivant l'ouverture de la procédure de collecte des signatures. Le résultat du référendum lie les autorités à la condition qu'au moins la moitié des électeurs inscrits se soit prononcée. Le World Macedonian Congress (Svetskiot makedonski kongres), une ONG dirigée par l'ancien député Todor Petrov, est parvenu à réunir 180454 signatures avec le soutien des partis d'opposition et de plusieurs ONG macédoniennes. Le 3 septembre 2004, le Parlement en a pris acte et a fixé la date du référendum au 7 novembre.
} 
populaire représentera sans nul doute un test décisif pour la poursuite des réformes. Dans cette situation de grande confusion politique, les élections locales, prévues à l'origine pour le 17 octobre, ont été reportées au printemps 2005.

À mi-parcours, quel bilan peut-on tirer des efforts pour donner vie aux principes d'Ohrid ? En 2001, les Occidentaux avaient vu dans les accords un prélude au rétablissement de la coexistence multiethnique. Quand bien même la restructuration de la gestion communale serait menée à bien, il n'est pas impossible que s'instaure plutôt une logique de développement séparé. La décentralisation, d'une part, la ségrégation de plus en plus marquée de l'éducation, d'autre part, signifient que les générations à venir auront de moins en moins l'opportunité de fréquenter les représentants d'une communauté différente de la leur. Il paraît difficile de déterminer aujourd'hui dans quelle mesure la tendance à la distanciation sera pérenne. Pour empêcher une telle évolution, le pays a besoin de temps et de paix sociale. Or les accords d'Ohrid n'avaient nullement vocation à traiter les maux socio-économiques susceptibles de fragiliser le tissu intercommunautaire. C'est ici l'articulation entre les volets économiques et politiques de l'action européenne, entre les mesures prises dans le cadre d'Ohrid et celles concernant la préparation de l'intégration à l'Union européenne, qui pose problème.

\section{L'hypothèque socio-économique de la paix : les exigences contradictoires de I'Union européenne et du Fonds monétaire international}

La Macédoine doit aujourd'hui affronter une crise socio-économique dont il n'est pas évident que l'Union européenne et, plus largement, la communauté internationale, mesurent toutes les implications politiques. Surtout, les recommandations adressées par les représentants de l'Union et de la communauté internationale aux autorités macédoniennes sont pétries de contradictions : recherche de stabilité politique et exigences macroéconomiques ne font pas nécessairement bon ménage. Ces contradictions trahissent un manque de coordination entre les acteurs impliqués dans la formulation des politiques européennes en Macédoine.

Depuis la création de l'État indépendant en septembre 1991, le PNB macédonien a chuté d'un quart. Le revenu moyen par habitant stagne à environ 2000 euros par an (2002)15. La crise a été aggravée par les retards pris dans les réformes structurelles : la privatisation, lancée en 1993, n’a connu d'accélération qu'en 2003. En 2003-2004, la restructuration des établissements publics a commencé à produire des effets bien connus des économies postsocialistes : en l'espace d'un an, le taux de chômage est passé de 31,9 \% à 36,7 \%, chiffre élevé, même si l'on tient compte

15. Cf. European Commission, « The Western Balkans in Transition », European Economy. Occasional Papers, 5, janvier 2004, p. 51. 
de l'existence d'une économie informelle. Face à ce marasme social, la communauté internationale envoie des signaux ambivalents. Elle parle de stabilisation tout en soutenant des politiques vouées à avoir des incidences désastreuses sur la qualité de vie d'une majorité des citoyens. Fidèle à lui-même, le Fonds monétaire international (FMI) a fait savoir à la Macédoine qu'elle consacrait une partie excessive de son budget à l'emploi public. L'accord de stand-by signé en avril 2003 a eu pour contrepartie une promesse de réduction du déficit budgétaire et de rationalisation de la fonction publique, terme pudique pour décrire des licenciements. En 2003, les autorités macédoniennes étaient parvenues à négocier un gel du nombre des employés d'État. Début 2004, il a cependant fallu annoncer la suppression de $4 \%$ des postes d'ici la fin de l'année. Or, dans le même temps, les accords d'Ohrid font obligation aux responsables macédoniens de poursuivre le processus de rééquilibrage au profit des communautés minoritaires... Associée à une redistribution des postes en faveur des Albanais, cette compression des effectifs est vouée à alimenter les ressentiments de la majorité slave.

La position adoptée par l'Union européenne sur ces questions n'est pas non plus dépourvue d'incohérences. L'Union figure parmi les principaux donneurs d'aide à la Macédoine : entre 1992 et 2003, le pays a reçu 677 millions d'euros. Depuis 2001, l'assistance à la Macédoine est principalement administrée dans le cadre du programme CARDS, un instrument de financement destiné aux Balkans de l'Ouest. À ce titre, 56,2 millions d'euros ont été alloués à la Macédoine en 2001, 36,5 en 2002 et 38,5 en 200316. Au titre du programme 2004, 22,5 millions devraient être consacrés aux questions économiques et sociales et 17,0 millions aux affaires intérieures et à la justice. Mais ces chiffres globaux masquent plusieurs limites de l'aide. La première tient aux délais qui séparent l'annonce d'un crédit de son déblocage effectif : au 10 juin 2004, seuls $71 \%$ des financements 2002 avaient été contractés et $31 \%$ effectivement versés (14\% et $3 \%$ respectivement pour le budget 2003$)^{17}$. La seconde concerne les conditions d'octroi des aides : l'attribution par l'Union européenne d'une assistance macro-financière au budget obéit en effet au même type de conditionnalité que le soutien du FMI (la préservation des grands équilibres macro-économiques et la réduction des dépenses publiques) ${ }^{18}$. L'Union européenne a pourtant souligné à plusieurs reprises son attachement à la cohésion sociale. De façon plus générale, l'arbitrage entre différentes conceptions de la rationalité économique n'est pas

16. Cf. http://europa.eu.int/comm/external_relations/see/fyrom/ (consulté le 3 mars 2004).

17. La même remarque s'applique à la décision, prise à Thessalonique, d'étendre aux États des Balkans de l'Ouest l'éligibilité à plusieurs programmes communautaires. La Commission estime que les pays concernés pourraient accéder progressivement à ces budgets à partir de l'année 2005. Cf. http://www.ear.eu.int/macedonia/macedonia.htm (consulté le 10 juin 2004). 18. Entre 1992 et 2003, l'Union européenne a fourni à la Macédoine une assistance macro-financière de 138 millions d'euros. Cf. Assen Slim, « L'aide occidentale à l'Europe de l'Est : les leçons d'un demi-siècle d'aide au développement ontelles été tirées? », Transitions, 2, 1998, p. 29-71. 
évident : dans l'aide aux PME, par exemple, faut-il privilégier les entreprises les mieux placées pour affronter la concurrence (souvent basées dans les capitales et relativement prospères), au risque d'aggraver les disparités entre le centre et la périphérie, ou doit-on soutenir des firmes opérant dans des régions défavorisées et des secteurs peu rentables (comme l'agriculture) ? C'est souvent la première option qui est privilégiée. De la même façon, les programmes sociaux soutenus par l'Agence européenne pour la reconstruction (AER) trahissent une vision assez néolibérale de l'économie : les consultants étrangers misent sur une « flexibilisation » du marché du travail, c'est-à-dire sur une suppression des barrières aux licenciements ${ }^{19}$.

Pire encore, d'un côté, l'Union européenne incite à l'augmentation des dépenses pour accompagner la mise en œuvre des accords d'Ohrid ; de l'autre, elle appelle à leur réduction. Le troisième rapport annuel sur le Processus de stabilisation et d'association, diffusé en mars 2004, fournit une bonne illustration de cette contradiction : tout en soulignant la nécessité de « prévoir une augmentation des ressources budgétaires de façon à atteindre progressivement les objectifs fixés dans l'accordcadre, de nouvelles actions de formation ciblées et des modifications des règlements sur l'emploi dans le secteur public » concernant la représentation équitable des minorités dans l'administration ${ }^{20}$, le rapport approuve la réduction du déficit budgétaire (ramené de 5,7 \% du PIB en 2002 à 1,5 \% en 2003). Et les auteurs feignent de s'étonner que les dépenses relatives à la décentralisation n'aient pas été proprement budgétées en 2004.

Plus fondamentalement, il semblerait que les divers « bras » de l'action européenne peinent à coordonner leurs interventions. En Macédoine, l'Union européenne s'incarne en effet dans au moins trois entités, l'Office du Représentant spécial, la Délégation de la Commission et l'Agence européenne pour la reconstruction. Le temps passé à aplanir les différends entre Européens semble parfois excéder celui consacré à tisser des liens avec les autorités locales. L'Office, en tant que responsable de la mise en œuvre des accords d'Ohrid, dispose d'une vision politique, mais son mandat est limité aux seuls accords (principalement des questions juridiques et institutionnelles déconnectées des aspects économiques et sociaux) et à une période circonscrite (jusqu'en 2005-2006). Qui plus est, il ne dispose pas d'un budget propre. La Délégation, elle, se concentre sur le suivi de l'Accord de stabilisation et d'association (ASA) et les perspectives d'intégration européenne, sans pouvoir incorporer dans cette vision les questions relatives à la gestion politique de la diversité intercommunautaire.

\footnotetext{
19. Entretien avec Dimitra Ioannou, consultante pour l'AER en charge de la rédaction du Plan d'action pour l'emploi, Skopje, 11 décembre 2003. Le rapport de la Commission européenne sur les Balkans de l'Ouest de janvier 2004 suggère lui aussi une « flexibilisation » du marché du travail. Cf. European Commission, « The Western Balkans in Transition », European Economy. Occasional Papers, 5, janvier 2004, p. 55.

20. Cf. Commission des communautés européennes, Document de travail des services de la Commission. Ancienne République yougoslave de Macédoine. Rapport de stabilisation et d'association - 2004. Bruxelles, Com (2004) 204 final, p. 5.
} 
Et elle non plus ne dispose pas de budgets conséquents. Enfin, l'Agence européenne pour la reconstruction administre les financements CARDS... mais les autres acteurs européens lui reprochent volontiers son manque de vision politique.

Avec la concrétisation progressive des perspectives d'adhésion à l'Union européenne de la Macédoine, une rationalisation de ces dispositifs pourrait être anticipée par le renforcement du rôle de la Délégation et des budgets d'aide à la transposition de l'acquis communautaire, ces quelque 80000 pages où sont définies les droits et obligations qui lient les États membres de l'Union européenne. Mais, là encore, reste à déterminer si la seule promesse européenne suffira à faire oublier la crise sociale et les tensions intercommunautaires et si les aides à l'intégration sont bien adaptées aux besoins de la Macédoine.

\section{Les perspectives d'intégration à l'Union européenne}

Le 21 juin 2003, le sommet de Thessalonique a confirmé la « perspective européenne » des Balkans de l'Ouest. Il a aussi prévu la mise en place de mécanismes qui ne sont pas sans rappeler ceux utilisés dans les relations avec les pays d'Europe centrale et orientale (tels les Partenariats européens, un monitoring sous forme de rapports annuels, les jumelages, etc.). Nombreux sont les analystes qui, ces dernières années, ont invité à relativiser la capacité de l'Union européenne à transformer les institutions des pays candidats ${ }^{21}$. En l'occurrence, la question ne se pose pas en ces termes : dans des États confrontés à une grave crise économique et sociale et à une fragilité identitaire certaine, elle porte plutôt sur la capacité du projet d'intégration à modifier les anticipations des acteurs politiques et à fermer la voie à toute tentation autoritaire ou violente. De ce point de vue, les exemples de la Bulgarie et de la Croatie, pour s'en tenir à des illustrations « balkaniques », sont très éloquents : à partir du moment où la perspective européenne a commencé à être crédible, elle a transformé le jeu politique. Tout « retour en arrière » autoritaire ou nationaliste extrême a été exclu de la gamme des possibles, tandis que la compétition partisane se réorganisait autour des espoirs euro-atlantiques.

Le 22 mars dernier, la Macédoine a déposé sa candidature à l'Union européenne. Cette date répondait à deux impératifs. Il s'agissait d'abord de se placer sur les rangs avant l'élargissement à vingt-cinq, les élections européennes de juin et, surtout, la formation d'une nouvelle Commission à l'automne. Le but était ensuite de montrer à une opinion publique plutôt sceptique que la perspective européenne pouvait prendre forme. Car aujourd'hui encore, en Macédoine, la majorité de la société doute de la viabilité d'un État commun. Depuis son arrivée

21. Cf. Heather Grabbe, « How Does Europeanization Affect CEE Governance ? Conditionality, Diffusion, and Diversity », Fournal of European Public Policy, 8 (6), 2001, p. 1013-1031. 
au pouvoir, le SDSM - qui fut relayé en cela par le défunt président, Boris Trajkovski, pourtant membre du parti concurrent, la VMRO-DPMNE - tente de convaincre que l'éclatement n'est pas inéluctable et que la double adhésion à l'OTAN et à l'Union européenne peut fournir une alternative à un scénario catastrophiste. La réponse positive apportée par la Commission européenne à la demande d'ouverture de pourparlers d'adhésion de la Croatie, le 20 avril 2004, constitue de ce point de vue une excellente nouvelle. Le dépôt de la candidature macédonienne contribue, avec la mise en place de groupes de travail en charge de l'intégration au sein des ministères et la multiplication des conférences sur les échanges d'expérience entre candidats à l'Union, à rendre visible l'option européenne auprès du public. Quelques effets en sont déjà perceptibles : depuis le mois de février, les médias établissent de plus en plus souvent des parallèles avec la Bulgarie et la Croatie tout en spéculant sur le futur calendrier d'adhésion.

Ce travail de conviction risque cependant de se heurter à trois obstacles. Premièrement, le désir de ménager un compromis intercommunautaire pour maximiser les chances d'intégration à l'Union européenne (et à l'OTAN) est principalement porté par le SDSM et le BDI, les deux forces politiques au pouvoir. Or l'expérience (de la Bulgarie notamment) a montré que les formations politiques qui administraient des réformes de structure extrêmement impopulaires au nom d'un objectif extérieur, devaient en payer tôt ou tard le prix. D'ores et déjà, les cotes de popularité du SDSM et du BDI au sein de leurs communautés respectives sont en chute. Les Albanais dénoncent les lenteurs dans la mise en œuvre des réformes, la crise de l'emploi et la persistance de pratiques corrompues. La majorité slave contemple, avec une exaspération grandissante, les concessions faites aux minorités et le démantèlement d'un secteur public où elle détenait l'essentiel des responsabilités. En Bulgarie, lorsque la crise sociale est devenue trop profonde, un homme politique a surgi pour rassembler les mécontents, l'ancien roi Siméon II, rentré d'exil. Il l'a fait sur un mode démagogique, mais a néanmoins poursuivi les réformes. Dans le contexte infiniment plus fragile de la Macédoine, le problème vient de l'absence d'opposition politique en mesure de fournir une alternative au SDSM-BDI tout en continuant à avancer sur la voie de l'Union européenne. À l'heure actuelle, en effet, la VMRO-DPMNE est déchirée entre une aile pragmatique, dirigée par Nikola Gruevski, ancien ministre des Finances, et une branche « dure » derrière l'ancien Premier ministre, Ljubč Georgievski, qui s'était distingué par des appels à la partition de la Macédoine en 2003. Côté albanais, la principale force d'opposition, le PDA d'Arben Xhaferi, a également tenté de jouer la carte de la radicalisation en brandissant le spectre d'une division territoriale. Le référendum sur le découpage territorial, prévu pour le 7 novembre 2004 et les élections municipales du printemps 2005 devraient fournir un observatoire crucial de l'évolution des rapports de force de ces deux formations. 
La seconde hypothèque tient à l'environnement régional de la Macédoine. Au moment des violences de mars 2004 au Kosovo, la peur de dérapages intercommunautaires est réapparue en Macédoine. Il s'agissait d'une crainte d'affrontements, non d'un désir d'en découdre. Les relations entre dynamiques politiques au Kosovo et en Macédoine n'ont sans doute pas le caractère de contagion automatique qu'on leur prête volontiers. Mais une déstabilisation durable du Kosovo n'en aurait pas moins des répercussions sur la Macédoine, ne serait-ce que parce que la majorité slave est convaincue que l'avenir du pays est suspendu à celui du Kosovo et que la déstabilisation est toujours importée.

Enfin, la troisième incertitude porte sur les instruments dont dispose l'Union européenne pour soutenir les réformes. Car les outils utilisés avec les dix pays entrants ne sont que partiellement adaptés aux besoins de la Macédoine. L'essentiel de l'assistance est de type juridique et institutionnel et vise à faciliter la reprise de l'acquis communautaire. Dans le contexte de la Macédoine, les problèmes rencontrés ne sont pas seulement, voire pas principalement, liés à la reprise de l'acquis, mais à la convergence entre crise de transformation et crise de mal-développement. Comme en Bosnie-Herzégovine, au Kosovo et dans certaines parties de la Serbie, l'industrialisation macédonienne date de la période communiste. Menée à marche forcée, elle a souvent pris la forme d'investissements peu rentables dont la vocation était au moins autant de stabiliser politiquement les zones concernées que de rationaliser la production. Les bouleversements des années 1990 ont remis en question ces « avancées ». Dès lors, se concentrer sur la seule transposition de l'acquis ne suffira pas à mettre le pays sur la voie d'une croissance durable, porteuse de dividendes sociaux et de sérénité intercommunautaire. Peut-être serait-il ici souhaitable de tirer quelques leçons des expériences centre-européennes : s'en remettre à la seule main invisible de la privatisation ne suffira pas à relancer l'économie. La conception d'une politique industrielle et de développement rural représente un complément indispensable.

C'est précisément dans la négligence des soubassements socio-économiques de la paix que se situe la principale faiblesse du dispositif européen actuel. Or la crise sociale constitue une bombe ethnique à retardement. Faute de s'en souvenir, les Européens pourraient bien voir la success story macédonienne se transformer en échec.

Nadège Ragaru est chercheur à l'Institut de relations internationales et stratégiques (IRIS), en charge du Programme "Europe centrale et orientale ", et enseigne à l'Institut d'études politiques (IEP) de Lille. Ses recherches portent sur la gestion des crises dans les Balkans, l'élargissement de l'EU aux États d'Europe du Sud-Est et les réseaux politiques en Bulgarie. Elle a dernièrement publié "Bulgarie : les couleurs de la différence ", dans Patrick Michel (dir.), Europe centrale : la mélancolie du réel (Paris, CERI/Autrement, 2004) et "Uslugi: The Role of Political Favors and Connections in Post-Communist Bulgaria ", dans Dimitris Keridis, Ellen Elias-Bursac, Nicholas Yatromanolakis, New Approaches to South-East European Studies (Herndon, Brassey's \& IFPA-Kokkalis Series on Southeast European Policy, 2003). E-mail : nragaru@yahoo.fr 